\title{
Predicción del efecto de cultivares de algodón en la presencia de interacción genotipo-ambiente
}

\author{
Freddy Mora ${ }^{1}$, Osmério Pupim-Junior ${ }^{2}$ y Carlos Alberto Scapim ${ }^{1}$ \\ ${ }^{1}$ Departamento de Agronomía. Centro de Ciencias Agrarias - UEM. \\ Rua Santos Dumont 2353-A, Apto. 301, Zona 01, 87013-050. Maringá, Paraná, Brasil. \\ ${ }^{2}$ Cooperativa Central Agropecuaria de Desarrollo Tecnológico y Económico, COODETEC. \\ Estado de Paraná, Brasil.
}

\begin{abstract}
F. Mora, O. Pupim-Junior, and C.A. Scapim. 2007. Prediction of cultivar effects on cotton yield in the presence of genotype-environment interaction. Cien. Inv. Agr. 34(1):13-21. We aimed to obtain, by the Best Linear Unbiased Predictor (BLUP), a prediction of the cultivar effect of 14 cotton cultivars (Gossypium hirsutum) established at different sites in Brazil and Paraguay during the 2003-2004 growing season. The BLUP of cotton yield was compared with classical stability analysis according to the Plaisted and Peterson, Wricke, Annicchiarico and Lin and Binns models. The Independence Chain (IC) algorithm, within a Bayesian framework, was also utilized for comparison. A likelihood ratio test provided evidence of significant differences for cultivar and genotype-environment interaction effects. These results were confirmed by Akaike and Bayesian information criteria. Most Spearman and Pearson correlation values were not significant site-to-site ( $\mathrm{p}>0.05$ ), varying from $-0.037 \pm 0.307$ to $0.565 \pm 0.290$ and from $-0.228 \pm 0.336$ to $0.604 \pm 0.257$, respectively. The Plaisted and Peterson and Wricke methods showed no significant correlation with BLUP, IC, Annicchiarico and Lin and Binns. On the other hand, Spearman rank coefficients were high and significant $(\mathrm{p}<0.01)$ between BLUP and Annicchiarico $(0.859 \pm 0.151)$ and BLUP and Lin and Binns $(-0.899 \pm 0.099)$. BLUP and IC, based on mean and median posterior estimates, had identical ranks. CD-406 and CD992221 were the cultivars that evidenced superior stability, as confirmed equally by BLUP, IC, Annicchiarico and Lin and Binns, indicating a significant concordance between approaches in relation to genotypic stability. A mixed linear models methodology allows us to get important genotypic information of cotton cultivars with both high productivity and stability in the environments where they will be cultivated by farmers.
\end{abstract}

Key words: BLUP, likelihood, genotype-environment interaction, Gossypium hirsutum, improvement, cultivar stability.

\section{Introducción}

El algodón (Gossypium hirsutum L.), es uno de los productos agrícolas más importantes de la civilización moderna, destacándose por su complejidad en los procesos de producción e industrialización, y por la elevada utilización de mano de obra. El algodón es una de las fibras vegetales cultivadas más antiguas del mundo. Las primeras referencias registran su cultivo

Recibido 01 de agosto 2006. Aceptado 20 de diciembre 2006 'Dirigir correspondencia a F. Mora: fmora@universiabrasil.net algunos siglos antes de Cristo. En Brasil, las referencias sobre su historia son escasas; sin embargo, existen antecedentes de su existencia en la época del descubrimiento. Aparentemente, pueblos indígenas lo cultivaban para producir hilos y tejidos (Canechio-Filho et al., 1972).

En Brasil, el cultivo de algodón tiene gran importanciacomercial.Secultivaprincipalmente en secano, en una gran variedad de condiciones agro-ecológicas, y con diferentes niveles tecnológicos que varían entre la agricultura familiar hasta empresas altamente tecnificadas del Centro-Oeste del país. Además, se cultiva en 
condiciones semiáridas del Nordeste brasileño.

Las empresas proveedoras de semillas de algodón deben garantizar altos rendimientos en fibra, y otras características comerciales, con genotipos identificados para el ambiente donde serán cultivados. En este sentido, la metodología de modelos lineales mixtos, utilizada ampliamente en el mejoramiento de otras especies vegetales, puede aportar importantes evidencias sobre la calidad genética de los genotipos de algodón en estudio.

Piepho y Möhring (2006) discutieron la utilidad de modelos lineales mixtos en etapas avanzadas de selección de cultivares, como en ensayos de valor de cultivo y utilización (VCU), que consideran ensayos establecidos en diversos ambientes. En el estudio realizado por estos investigadores se demostró que la máxima verosimilitud restringida (REML: Restricted Maximum Likelihood) fue preferible a la máxima verosimilitud (ML), en la estimación de componentes de varianza. A su vez en ensayos VCU, la mejor predicción linear no sesgada (BLUP) es preferible al mejor estimador linear no sesgado (BLUE).

Tradicionalmente en evaluación de cultivares, el efecto debido al cultivar se ha considerado como un factor fijo (Piepho y Möhring, 2006). Tal efecto se ha reportado como aleatorio en la literatura, posibilitando la estimación de componentes de varianza de cultivares, la obtención de valores genéticos, y la utilización de la metodología de modelos lineares mixtos (Resende et al., 2001; Astorga y Mora, 2005; Piepho y Möhring, 2006).

Para la estimación de los efectos fijos y la obtención de valores genéticos predichos, se deben conocer y estimar con exactitud los componentes de varianza. Diversos trabajos han demostrado que el procedimiento REML, es actualmente el método adoptado para la obtención de BLUE y BLUP en modelos lineales mixtos (Mora y Arnhold, 2006). Si los valores de los componentes de varianza son conocidos, la predicción de valores genéticos vía BLUP se puede obtener utilizando las ecuaciones del modelo mixto (Henderson, 1973 y 1984).
Por otra parte, el conocimiento de la magnitud de la interacción entre el genotipo y el ambiente permite evaluar la estabilidad de los cultivares en una gama de ambientes donde se desean introducir. También permite evaluar los potenciales productivos y las posibles limitaciones de éstos en cada localidad (Contreras y Krarup, 2000). La interacción de cultivares con los factores ambientales (sitios, año de plantación, tipo de suelo, nivel de tecnología usado, etc.) es de gran importancia para los agricultores y en los programas de mejoramiento de plantas. Los efectos que los cultivares y el ambiente ejercen sobre la interacción cultivar-ambiente son estadísticamente no aditivos. Esto indicaría que las diferencias en productividad entre cultivares dependerá del ambiente en el cual están siendo cultivados (Yue et al., 1997; Scapim et al. 2000; Contreras y Krarup, 2000).

El objetivo de este trabajo fue analizar la predicción del efecto de cultivar, para el rendimiento de algodón, utilizando la mejor predicción linear no sesgada, comparando los valores predichos con los análisis clásicos de estabilidad de los modelos de Plaisted y Peterson (1959), Wricke (1965), Annicchiarico (1992), y no paramétrico de Lin y Binns (1988). Adicionalmente, se utilizó una estrategia Bayesiana, a través del algoritmo de cadena independiente, para la predicción del efecto de cultivar.

\section{Materiales y métodos}

Se analizó el rendimiento de 14 cultivares de algodón (CD-401, CD-406, CD-407, IPR120, CD98-1160, CD98-1304, CD99-2221, CD99-2225, CD00-580, CD00-628, CD00810, CD00-945, CD00-1160 y CD00-1390) en diferentes localidades de Brasil y Paraguay. En Brasil, los sitios correspondieron a las localidades de Palotina ( $24^{\circ} 17^{\prime}$ Lat. S.; $53^{\circ} 50^{\prime}$ Long. O.; $290 \mathrm{~m}$ de altitud) y Ubiratã (24³2' Lat. S.; 52 59' Long. O.; $550 \mathrm{~m}$ de altitud), del Estado de Paraná, y a la localidad de Ituverava $\left(20^{\circ} 20^{\prime}\right.$ Lat. S.; 47\%46' Long. O.; $620 \mathrm{~m}$ de altitud), del Estado de San Pablo. En Paraguay, el sitio de estudio correspondió a la localidad de Maria Auxiliadora (242ㅇ' Lat. S.; 56 $30^{\circ}$ ' Long. O.; $250 \mathrm{~m}$ de altitud). Se utilizó el diseño 
de bloques completos al azar (BCA), con cuatro repeticiones, como diseño experimental. Las mediciones del rendimiento de algodón se realizaron en la temporada agrícola 2003-2004.

Se utilizó el siguiente modelo estadístico, que incluyó factores fijos y aleatorios, de acuerdo a la metodología de modelos mixtos definida por Henderson $(1973,1984)$ :

$$
y=X \beta+Z_{1} \mu+Z_{2} w+\varepsilon
$$

donde $y$ es el vector de observaciones (rendimiento de cultivares de algodón); $X$ es la matriz de incidencia de los efectos fijos; $\beta$ es el vector del efecto fijo debido al sitio y al bloque; $Z_{1}$ es la matriz de incidencia de los efectos aleatorios; $\mu$ es el vector de los efectos aleatorios de cultivares; $w$ corresponde a la interacción cultivar-sitio; $\varepsilon$ es el vector aleatorio de residuos. Se consideró que las observaciones y los efectos aleatorios siguen la siguiente distribución normal multivariada:

$$
\left[\begin{array}{c}
y \\
\mu \\
w \\
\varepsilon
\end{array}\right] \sim N M V\left(\left[\begin{array}{c}
X \beta \\
0 \\
0 \\
0
\end{array}\right],\left[\begin{array}{cccc}
V & Z_{1} G & Z_{2} W & R \\
G Z_{1}^{\prime} & G & 0 & 0 \\
W Z_{2} & 0 & W & 0 \\
R & 0 & 0 & R
\end{array}\right]\right)
$$

donde 0 es el vector nulo; $\emptyset$ es la matriz nula; $\mathrm{G}$ es la matriz de varianzas inter-genotípicas; $\mathrm{W}$ es la matriz de varianza de la interacción; $R$ es la matriz de varianzas residuales. Considerando el modelo (1), el Mejor Estimador Linear no Sesgado (BLUE) para $\beta$ y la Mejor Predicción Lineal no Sesgada (BLUP) para los efectos aleatorios $\mu$ y $w$ son dados por la siguiente ecuación de modelos mixtos (Henderson, 1973, 1984):

$$
\left[\begin{array}{ccc}
X^{\prime} R^{-1} X & X^{\prime} R^{-1} Z_{1} & X^{\prime} R^{-1} Z_{2} \\
Z_{1}^{\prime} R^{-1} X & Z_{1}^{\prime} R^{-1} Z_{1}+G^{-1} & Z_{1}^{\prime} R^{-1} Z_{2} \\
Z_{2}^{\prime} R^{-1} X & Z_{2}^{\prime} R^{-1} Z_{1} & Z_{2}^{\prime} R^{-1} Z_{2}+W^{-1}
\end{array}\right]\left[\begin{array}{c}
\beta \\
\mu \\
w
\end{array}\right]=\left[\begin{array}{c}
X^{\prime} R^{-1} y \\
Z_{1}^{\prime} R^{-1} y \\
Z_{2}^{\prime} R^{-1} y
\end{array}\right]
$$

Si los valores de los componentes de varianza son conocidos, la predicción de valores genéticos, vía la mejor predicción lineal no sesgada, puede ser obtenida utilizando las ecuaciones del modelo mixto (Henderson, 1973 y 1984; Perri y Iemma, 1999; Martins et al. 1997). Si tales componentes de varianza son desconocidos, y si estimados, la mejor predicción lineal no sesgada de los valores genéticos y la mejor estimación lineal no sesgada de los efectos fijos serían empíricos, EBLUP (empirical best linear unbiased preditor) y EBLUE (empirical best linear unbiased estimator), respectivamente (Littell et al., 1996).

Las estimaciones de componentes de varianza y las soluciones de los efectos aleatorios se calcularon en un único proceso iterativo de estimación-predicción, utilizando el procedimiento MIXED (Mixed Linear Model) del software SAS (SAS Institute, NC, EUA, 1996). Se utilizaron las opciones SOLUTION y REML (máxima verosimilitud restringida) para la obtención de EBLUP y componentes de varianza, respectivamente (Mora y Arnhold, 2006)

En el método REML, la función de verosimilitud es dividida en dos partes independientes: efectos fijos y aleatorios. Esta metodología implementada en el PROC MIXED de SAS utiliza un algoritmo de Newton-Raphson, iterando hasta dar convergencia a la función objetivo (logaritmo de la verosimilitud) de la parte que no contiene efectos fijos. El procedimiento MIXED utiliza varianzas previamente estimadas para valores iniciales de la convergencia, que son estimadas a través del método del estimador cuadrático no sesgado de varianza mínima (MIVQUE). Las ecuaciones REML para la estimación de componentes de varianza se pueden expresar de la siguiente manera (4):

$$
\left[\begin{array}{ccc}
\operatorname{tr}\left(V^{-1} Z_{1} G Z_{1}^{\prime} V^{-1} Z_{1} G Z_{1}^{\prime}\right) & \operatorname{tr}\left(V^{-1} Z_{1} G Z_{1}^{\prime} V^{-1} Z_{2} Z_{2}^{\prime}\right) & \operatorname{tr}\left(V^{-1} Z_{1} G Z_{1}^{\prime} V^{-1}\right) \\
\operatorname{tr}\left(V^{-1} Z_{2} W Z^{\prime}{ }_{2} V^{-1} Z_{1} G Z^{\prime}{ }_{1}\right) & \operatorname{tr}\left(V^{-1} Z_{2} W Z_{2}{ }_{2} V^{-1} Z_{2} Z_{2}\right) & \operatorname{tr}\left(V^{-1} Z_{2} W Z^{\prime}{ }_{2} V^{-1}\right) \\
\operatorname{tr}\left(V^{-1} V^{-1} Z_{1} G Z^{\prime}{ }_{1}\right) & \operatorname{tr}\left(V^{-1} V^{-1} Z_{2} Z_{2}^{\prime}\right) & t\left(V^{-1} V^{-1}\right)
\end{array}\right]\left[\begin{array}{c}
\sigma_{c}^{2} \\
\sigma_{w}^{2} \\
\sigma_{\varepsilon}^{2}
\end{array}\right]=\left[\begin{array}{c}
y^{\prime} P Z{ }_{1} G Z_{1}^{\prime} P Z \\
y^{\prime} P Z_{2} W Z_{2} P Z \\
y^{\prime} P y
\end{array}\right]
$$


donde, $t r$ denota la traza de la matriz. $P$ es el proyector ortogonal para la parte aleatoria. $\sigma_{c}^{2}$, $\sigma_{w}^{2}$ y $\sigma_{\varepsilon}^{2}$ son los componentes de varianza del efecto de cultivar, interacción y residual, respectivamente.

Con el propósito de determinar la presencia de homogeneidad de varianzas residuales de cada sitio, requisito del análisis de varianza conjunta, se utilizó el comando REPEATED con la opción GROUP, en PROC MIXED (SAS Institute, 1996). Esta alternativa definió el efecto en el cual se especifica heterogeneidad en la estructura de co-varianza de la matriz R. Para comparar con el modelo homogéneo, se utilizó el procedimiento MIXED nuevamente pero en ausencia del comando REPEATED, dando origen a un análisis del modelo con varianzas homogéneas. Entonces se compararon los dos modelos, usando conjuntamente los criterios de información Bayesiano de Schwarz (BIC) y Akaike (AIC), así como el estadístico de la razón de verosimilitud (LRT: Likelihood Ratio Test). BIC, AIC y LRT poseen las siguientes ecuaciones:

$$
\begin{aligned}
& B I C=-2 \log R L+d \log n \\
& A I C=-2 \log R L+2 d \\
& L R T=2\left(\log R L_{+}-\log R L_{-}\right)
\end{aligned}
$$

donde $R L$ representa la máxima verosimilitud restringida; $R L_{+}$y $R L_{-}$son los valores máximos de la verosimilitud restringida de los modelos heterogéneos y homogéneos, respectivamente; $d$ es la dimensión del modelo; $n$ es igual al número de observaciones válidas para la estimación de REML.

El mismo procedimiento anterior fue también utilizado para verificar la presencia de interacción genotipo-ambiente, utilizando el estadístico de la razón de verosimilitud (Yang, 2002).

Como medida de la magnitud de la interacción genotipo-ambiente, o cultivar-sitio, las soluciones de los efectos aleatorios de la interacción (BLUP) se examinaron a través de la correlación de Pearson. Coeficiente de correlación de Spearman también se estimó como una medida de correlación entre los ranking por sitio (Scapim et al., 2000). Los errores estándar de cada valor de correlación se obtuvieron a través del método Bootstrap con 1000 muestras aleatorias. Los procedimientos de SAS: SURVEYSELECT, CORR y UNIVARIATE se utilizaron para crear y analizar los re-muestreos con reemplazo.

Adicionalmente, algunas medidas de estabilidad de los genotipos se realizaron para comparar tales valores con los obtenidos de BLUP. Los métodos utilizados para evaluar estabilidad fueron los propuestos por Plaisted y Peterson (1959), Wricke (1965), Lin y Binns (1988) y Annicchiarico (1992). Todos estos análisis se realizaron utilizando el programa estadístico GENES (disponible en www.ufv.br/dbg/genes/ genes.htm) (Cruz, 2001).

Una estrategia Bayesiana, a través del algoritmo de cadena independiente, derivada de los métodos de Monte Carlo vía Cadenas de Marcov, se utilizó como otra medida de comparación para la predicción del efecto del cultivar. Las estimativas puntuales del promedio, mediana y moda a posteriori se obtuvieron a través del procedimiento PROC MIXED con la opción PRIOR (Mora y Arnhold, 2006).

La comparación entre las distintas clasificaciones de genotipos se realizó vía correlación de Spearman, con errores estándar vía Bootstrap, similar al procedimiento informado anteriormente.

\section{Resultados y discusión}

Los resultados de la prueba de razón de verosimilitud indicaron ausencia de heterogeneidad de varianzas ambientales, aceptándose la hipótesis de nulidad con probabilidad de $\chi^{2}$ superior a 5\% (Cuadro 1 ). Basado en esta misma prueba estadística, los efectos de interacción cultivar-sitio y cultivar fueron estadísticamente significativos ( $\mathrm{p}<0,01$ $\mathrm{y}<0,05$; respectivamente). Los valores inferiores de los criterios de información Bayesiano de Schwarz (BIC) y Akaike (AIC), coincidieron con la selección del modelo completo (considerando todos los efectos) con la homogeneidad de las varianzas residuales. 
Cuadro 1. Prueba de la razón de verosimilitud (restringida) (LRT), criterios de información de Akaike (AIC) y Bayesiano (BIC), y mejor estimador lineal no sesgado (BLUE) del rendimiento de cultivares de algodón por sitio.

Table 1. Likelihood ratio test (restricted) (LRT), Akaike (AIC) and Bayesian (BIC) information criteria for several models, and best linear unbiased estimator (BLUE) for yield of cotton cultivars per site.

\begin{tabular}{|c|c|c|c|c|c|c|c|c|c|c|}
\hline \multirow[t]{3}{*}{ Modelo $^{1}$} & \multirow[t]{3}{*}{ AIC } & \multirow[t]{3}{*}{$\mathrm{BIC}$} & \multirow[t]{3}{*}{ LRT } & \multirow[t]{3}{*}{$\mathrm{p}>\chi^{2}$} & \multicolumn{5}{|c|}{ Efecto fijo: Sitio } & \\
\hline & & & & & \multirow[t]{2}{*}{ valor-F } & \multirow[t]{2}{*}{$\mathrm{p}>\mathrm{F}$} & \multicolumn{4}{|c|}{ BLUE, Sitios ${ }^{2}, \mathrm{~kg} \cdot \mathrm{ha}^{-1}$} \\
\hline & & & & & & & $\mathrm{S}_{1}$ & $\mathrm{~S}_{2}$ & $\mathrm{~S}_{3}$ & $\mathrm{~S}_{4}$ \\
\hline $\mathrm{M}_{\mathrm{F}}$ & $3.561,1$ & $3.563,7$ & & & 39,57 & $<0,01$ & $5.847,2$ & $6.530,5$ & $6.867,3$ & $4.385,2$ \\
\hline $\mathrm{M}_{\mathrm{FH}}$ & $3.563,6$ & $3.568,1$ & 3,5 & $>0,05$ & 40,69 & $<0,01$ & & & & \\
\hline $\mathrm{M}_{\mathrm{S} / \mathrm{I}}$ & $3.570,7$ & $3.572,6$ & 11,6 & $<0,01$ & 56,43 & $<0,01$ & & & & \\
\hline $\mathrm{M}_{\mathrm{S} / \mathrm{C}}$ & $3.563,8$ & $3.569,8$ & 4,7 & $<0,05$ & 32,87 & $<0,01$ & & & & \\
\hline
\end{tabular}

${ }^{1} \mathrm{M}_{\mathrm{F}}$ y $\mathrm{M}_{\mathrm{FH}}$ : modelos completos considerando homogeneidad y heterogeneidad de varianzas residuales; $\mathrm{M}_{\mathrm{S} / \mathrm{I}} \mathrm{y} \mathrm{M}_{\mathrm{S} / \mathrm{C}}$ : Modelos con homogeneidad de varianzas sin interacción y sin efecto de cultivar.

${ }^{2} \mathrm{~S}_{1}$ : Palotina; $\mathrm{S}_{2}$ : Ubiratã; $\mathrm{S}_{3}$ : Ituverava; $\mathrm{S}_{4}$ : Maria Auxiliadora.

${ }^{1} M_{H}$ and $M_{F H}$ : full models considering homogeneity and heterogeneity of residual variances; $M_{S / l}$ and $M_{S / C}:$ Models with homogeneity of variances without interaction and without cultivar effect.

${ }^{2} S_{1}$ : Palotina; $S_{2}:$ Ubiratã $; S_{3}$ : Ituverava; $S_{4}$ : Maria Auxiliadora.

El análisis de varianza convencional fue coincidente estadísticamente con los resultados del estadístico de la razón de verosimilitud, en todas las fuentes de variación (datos no mostrados).

El coeficiente de variación experimental fue bajo $\mathrm{CV}=10,9 \%$, e inferior al promedio encontrado por Moresco (2003), quien analizó diversos experimentos de $G$. hirsutum, en un periodo de 12 años de investigación en el Estado de Mato Grosso, Brasil. Para la productividad de algodón, este autor estimó coeficientes de variación entre 4,7 y 31,5\%, con un promedio de $14,3 \%$.

El efecto sitio fue estadísticamente significativo, según el análisis de los efectos fijos (Tipo 3) del procedimiento MIXED (SAS Institute, 1996). Este resultado fue invariante en relación al modelo en evaluación (Cuadro 1). Las estimaciones de los valores BLUE se muestran en el Cuadro 1 para cada sitio. Estos valores variaron de 4.385 a $6.867 \mathrm{~kg} \cdot \mathrm{ha}^{-1}$, correspondientes a las localidades de Maria Auxiliadora (Paraguay) e Ituverava (Estado de San Pablo, Brasil), respectivamente.

La predicción genética del efecto de cultivar para el rendimiento de algodón se muestra en el Cuadro 2. Entre sitios, algunos cultivares no mantuvieron su superioridad en rendimiento, confirmando la presencia significativa de interacción cultivar-sitio. Por ejemplo, el cultivar
CD00-810 presentó un valor predicho superior en el sitio de Palotina, pero esta superioridad no permaneció en los sitios de Ubiratã e Ituverava, evidenciando valores genéticos negativos y claramente inferiores al resto. También el cultivar IPR-120, mostró un BLUP superior en Ituverava $(938,0)$ pero negativo e inferior en Maria Auxiliadora $(-704,1)$. En estos dos sitios los valores del coeficiente de correlación de Spearman y Pearson fueron bajos y no significativos ( $p>0,05)$ (Cuadro 3). Esto sugiere una alta magnitud de la interacción genotipoambiente. El cultivar CD-406 mantuvo valores altos y positivos en todos los sitios, mostrando una cierta estabilidad en la predicción del rendimiento. La mayoría de los valores de correlación de Spearman y Pearson fueron no significativos estadísticamente $(\mathrm{p}>0,05)$.

En relación con la clasificación de cultivares basado en su valor de estabilidad y considerando todos los ambientes, los coeficientes de correlación de Spearman fueron mayores entre la clasificación vía BLUP y los modelos de Annicchiarico (1992) y Lin y Binns (1988) (Cuadro 4 y 5). Estos valores fueron estadísticamente significativos ( $\mathrm{p}<0,01)$ y correspondieron a ( \pm error estándar): $0,859 \pm 0,151$ y $-0,899 \pm 0,099$, respectivamente. Estos coeficientes de correlación significativos indican que los genotipos se ordenan indistintamente al método propuesto, o que la clasificación (ranking) de los cultivares no varía significativamente entre los distintos métodos. 
Cuadro 2. Resultados de la predicción del efecto de cultivar de algodón a través de la mejor predicción linear no sesgada (BLUP), obtenido por cada sitio.

Table 2. Results of the prediction of cotton cultivar effect by best linear unbiased predictor (BLUP), obtained per site.

\begin{tabular}{|c|c|c|c|c|}
\hline \multirow{2}{*}{$\begin{array}{l}\text { Cultivares de } \\
\text { Algodón }\end{array}$} & \multicolumn{4}{|c|}{ Localidades } \\
\hline & Palotina & Ubiratã & Ituverava & Maria Auxiliadora \\
\hline CD-401 & $-809,6$ & $-461,4$ & $-224,0$ & 45,8 \\
\hline CD-406 & 498,4 & 796,3 & 542,7 & 433,1 \\
\hline CD-407 & 170,5 & 431,7 & 83,4 & 2,4 \\
\hline IPR-120 & 162,7 & 505,8 & 938,0 & $-704,1$ \\
\hline CD98-1160 & $-315,2$ & $-225,3$ & $-21,7$ & 199,9 \\
\hline CD98-1304 & 30,0 & $-161,1$ & 30,0 & $-73,9$ \\
\hline CD99-2221 & 291,8 & 415,7 & 277,2 & 120,7 \\
\hline CD99-2225 & 138,7 & 10,6 & $-114,1$ & 213,9 \\
\hline CD00-580 & 21,8 & 254,8 & $-432,9$ & $-27,2$ \\
\hline CD00-628 & $-521,1$ & $-609,6$ & $-488,7$ & $-200,4$ \\
\hline CD00-810 & 505,5 & $-517,8$ & $-336,1$ & 60,9 \\
\hline CD00-945 & $-168,9$ & $-674,0$ & 284,1 & 39,1 \\
\hline CD00-1160 & $-350,9$ & $-58,8$ & $-292,2$ & $-62,2$ \\
\hline CD00-1390 & 346,3 & 293,0 & $-245,6$ & $-48,0$ \\
\hline
\end{tabular}

BLUP y el algoritmo de cadena independiente (IC), basado en las estimativas puntuales del promedio y la mediana a posteriori, tuvieron idénticos ordenamientos de los cultivares, confirmando lo reportado por Mora y Arnhold (2006) en la predicción del efecto familiar de genotipos de maíz. La estimativa de la moda a posteriori tuvo una pequeña diferencia en el ordenamiento de los cultivares CD98-1160 y CD00-945, aunque el valor de correlación de Spearman entre la moda IC y BLUP fue también cercano a 1 . Debido a la alta correlación entre IC y BLUP, la estimativa del promedio a posteriori, de cada valor predicho, tuvo una correlación idéntica a lo encontrado entre BLUP y los demás métodos de estabilidad (Cuadro 5).

En el procedimiento alternativo para evaluar el comportamiento de genotipos bajo interacción genotipo-ambiente propuesto por Lin y Binns (1988), la superioridad de un genotipo puede ser medida por el índice de superioridad $\left(\mathrm{P}_{\mathrm{i}}\right)$, el cual es definido como el desvío del i-ésimo cultivar relativo al genotipo que entrega la respuesta máxima en cada ambiente. Así, el genotipo superior debería ser aquel con menor valor $\mathrm{P}_{\mathrm{i}}$, el que permanecería dentro de los más productivos en un conjunto de ambientes de interés (Scapim et al., 2000; Elias et al., 2005). El cultivar CD-406 presentó el menor valor de $\mathrm{P}_{\mathrm{i}}$, coincidiendo con el mayor valor predicho por BLUP y algoritmo IC (Cuadro 4). Este cultivar debería recibir mayor atención del punto de vista de la superioridad promedio en los ambientes evaluados. A su vez, cabe destacar que este cultivar fue el único que mantuvo valores positivos (entre moderados y altos) de

Cuadro 3. Coeficientes de correlación de Spearman (mostrado en itálico) y correlación de Pearson calculados entre los sitios.

Table 3. Spearman's rank correlation coefficients (shown in italic) and Pearson correlations calculated among sites.

\begin{tabular}{|c|c|c|c|c|}
\hline & Palotina & Ubiratã & Ituverava & Maria Auxiliadora \\
\hline Palotina & --- & $0,565 \pm 0,259^{* 1}$ & $0,323 \pm 0,290 n s^{2}$ & $0,301 \pm 0,260 n s^{2}$ \\
\hline Ubiratã & $0,604 \pm 0,257^{* 1}$ & --- & $0,486 \pm 0,289 n s^{2}$ & $-0,037 \pm 0,307 n s^{2}$ \\
\hline Ituverava & $0,364 \pm 0,281 \mathrm{~ns}^{2}$ & $0,529 \pm 0,294 n s^{2}$ & --- & $0,213 \pm 0,326 n s^{2}$ \\
\hline Maria Auxiliadora & $0,153 \pm 0,264 \mathrm{~ns}^{2}$ & $-0,122 \pm 0,316 \mathrm{~ns}^{2}$ & $-0,228 \pm 0,336 \mathrm{~ns}^{2}$ & -- \\
\hline
\end{tabular}

${ }^{1}$ Correlación fue significativa $(\mathrm{p}<0,05)$ según t-student. ${ }^{2} \mathrm{~ns}$ : Correlación no fue significativa $(\mathrm{p}>0,05) . \pm$, error estándar.

${ }^{1}$ Correlation was significant $(p<0.05)$ according to Student's t-test. ${ }^{2} n s$ : correlation was not significant $(p>0.05) . \pm$, standard error. 
Cuadro 4. Predicción del efecto de cultivares de algodón (productividad) según mejor predicción linear no sesgada (BLUP), algoritmo de cadena independiente y parámetros de estabilidad de Plaisted y Peterson (1959), Wricke (1965), Annicchiarico (1992) y Lin y Binns (1988).

Table 4. Prediction of cultivar effect (yield) based on best linear unbiased prediction (BLUP), independence chain algorithm, and Plaisted and Peterson (1959), Wricke (1965), Annicchiarico (1992) and Lin and Binns (1988) stability parameters.

\begin{tabular}{|c|c|c|c|c|c|c|c|c|}
\hline \multirow{2}{*}{$\begin{array}{l}\text { Cultivares } \\
\text { de } \\
\text { Algodón }\end{array}$} & \multirow{2}{*}{ BLUP } & \multicolumn{3}{|c|}{ Cadena Independiente } & \multirow{2}{*}{$\begin{array}{l}\text { Plaisted y } \\
\text { Peterson }\end{array}$} & \multirow{2}{*}{ Wricke } & \multirow{2}{*}{ Annicchiarico } & \multirow{2}{*}{$\begin{array}{l}\text { Lin y } \\
\text { Binns }\end{array}$} \\
\hline & & Promedio & Mediana & Moda & & & & \\
\hline CD-401 & $-319,0$ & $-313,2$ & $-307,4$ & $-318,3$ & $192.493,5$ & $4.021 .898,7$ & 88,7 & $1.281 .202,6$ \\
\hline CD-406 & 506,7 & 500,9 & 497,8 & 514,9 & $20.586,1$ & $190.820,3$ & 113,8 & $39.110,9$ \\
\hline CD-407 & 143,2 & 140,8 & 133,7 & 117,8 & $43.169,0$ & $694.095,3$ & 102,7 & $321.433,7$ \\
\hline IPR-120 & 146,5 & 142,0 & 140,2 & 143,1 & $662.675,9$ & $14.500 .248,8$ & 95,5 & $578.256,8$ \\
\hline CD98-1160 & $-70,0$ & $-71,7$ & $-74,7$ & $-117,5$ & $86.356,2$ & $1.656 .553,3$ & 96,7 & $685.988,8$ \\
\hline CD98-1304 & $-37,7$ & $-36,4$ & $-34,4$ & $-19,9$ & $20.915,2$ & $198.153,3$ & 98,2 & $574.686,1$ \\
\hline CD99-2221 & 242,3 & 241,0 & 236,4 & 235,0 & $22.258,3$ & $228.085,2$ & 106,3 & $193.705,7$ \\
\hline CD99-2225 & 68,8 & 67,9 & 66,5 & 67,2 & $42.685,6$ & $683.322,2$ & 101,3 & $468.895,0$ \\
\hline CD00-580 & $-44,5$ & $-43,8$ & $-42,5$ & $-43,3$ & $95.645,2$ & $1.863 .564,5$ & 97,3 & $692.033,1$ \\
\hline CD00-628 & $-401,5$ & $-394,3$ & $-391,0$ & $-394,0$ & $36.569,8$ & $547.026,9$ & 88,1 & $1.422 .067,5$ \\
\hline CD00-810 & $-34,6$ & $-33,0$ & $-31,6$ & $-16,4$ & $249.962,6$ & $5.302 .637,6$ & 96,8 & $824.793,4$ \\
\hline CD00-945 & $-100,7$ & $-101,4$ & $-100,8$ & $-115,2$ & $171.713,9$ & $3.558 .811,0$ & 95,1 & $768.390,0$ \\
\hline CD00-1160 & $-174,4$ & $-172,1$ & $-166,1$ & $-154,0$ & $41.732,8$ & $662.089,3$ & 94,1 & $864.954,0$ \\
\hline CD00-1390 & 74,8 & 76,6 & 77,5 & 103,9 & $104.582,0$ & $2.062 .727,5$ & 100,2 & $505.478,0$ \\
\hline
\end{tabular}

los valores genéticos predichos vía BLUP, a través de todos los sitios considerados (Cuadro 2). Además, en el modelo de Annicchiarico (1992) también presentó el comportamiento superior con un valor de 113,8. En general, BLUP, IC, Annicchiarico (1992), y Lin y Binns (1988) coincidieron en que los cultivares CD406 y CD99-2221 evidencian mayor estabilidad en los sitios considerados.

Scapim et al. (2000) sostiene que la metodología de Lin y Binns (1988) puede ser una buena alternativa para evaluar el rendimiento de un cultivar en la presencia de interacción genotipoambiente. Tal método no tendría limitaciones inherentes al uso de regresión. La definición de superioridad es similar a las definidas en los objetivos de un programa de mejoramiento, ya que un cultivar superior debería estar entre los más productivos en el mayor número posible de ambientes (Farias et al., 1997; Scapim et al., 2000).

Los métodos de Annicchiarico y de Lin-Binns presentaron una alta y significativa correlación de Spearman $(-0,947 ; \mathrm{p}<0,01)$, aspecto ya citado en la literatura (Artroch et al., 2000) debido a su cercana relación metodológica. A su vez, los métodos de Plaisted y Peterson (1959) y Wricke (1965) mostraron un coeficiente de Spearman

Cuadro 5. Coeficientes de correlación de Spearman calculados entre los procedimientos.

Table 5. Spearman's rank correlation coefficients calculated among procedures.

\begin{tabular}{|c|c|c|c|c|c|c|}
\hline & BLUP $^{1}$ & $\mathrm{IC}^{2}$ & Plaisted-Peterson & Wricke & Annicchiarico & Lin-Binns \\
\hline BLUP $^{1}$ & --- & $1,00 \pm 0,00^{* 3}$ & $-0,160 \pm 0,317 \mathrm{~ns}^{4}$ & $-0,160 \pm 0,324 n s^{4}$ & $0,859 \pm 0,151^{* 3}$ & $-0,899 \pm 0,099^{* 3}$ \\
\hline $\mathrm{IC}^{2}$ & & --- & $-0,160 \pm 0,319 n s^{4}$ & $-0,160 \pm 0,324 n s^{4}$ & $0,859 \pm 0,148^{* 3}$ & $-0,899 \pm 0,101^{* 3}$ \\
\hline Plaisted-Peterson & & & --- & $1,00 \pm 0,00^{* 3}$ & $-0,433 \pm 0,273 \mathrm{~ns}^{4}$ & $0,415 \pm 0,277 \mathrm{~ns}^{4}$ \\
\hline Wricke & & & & -- & $-0,433 \pm 0,264 \mathrm{~ns}^{4}$ & $0,415 \pm 0,279 \mathrm{~ns}^{4}$ \\
\hline Annicchiarico & & & & & --- & $-0,947 \pm 0,076^{* 3}$ \\
\hline Lin-Binns & & & & & & --- \\
\hline
\end{tabular}

'BLUP: mejor predicción linear no sesgada; ${ }^{2} \mathrm{IC}$ : algoritmo de cadena independiente basado en el promedio a posteriori; ${ }^{3}$ Correlación significativa $(\mathrm{p}<0,01)$ según $\mathrm{t}$-student; ${ }^{4} \mathrm{~ns}$ : Correlación no fue significativa $(\mathrm{p}>0,05)$. \pm , error estándar.

${ }^{1}$ BLUP: Best Linear Unbiased Predictor; ${ }^{2}$ IC: Independence Chain algorithm based on posterior mean; ${ }^{3}$ Correlation was significant $(p<0.01)$ according to Student's t-test; ${ }^{4} n s$ : Correlation was not significant $(p>0.05) . \pm$, standard error. 
con valor 1. Esto indicaría una perfecta correlación entre los ranking generados por estas metodologías, lo cual es confirmado con las correlaciones con los demás modelos. Esto ya ha sido ampliamente evidenciado en la literatura y se basa en el hecho de que ambos usan la descomposición de la suma de cuadrados del efecto de interacción genotipo-ambiente, en la derivación de sus parámetros de estabilidad (Cruz y Regazzi, 2001).

La predicción del efecto de cultivar en el análisis de interacción genotipo-ambiente en cultivares de algodón, permitió evidenciar cambios significativos en la respuesta de los cultivares a través de los ambientes. Consecuentemente, la utilización de coeficientes de Spearman y correlación de Pearson, permitieron determinar cambios en el ranking $u$ ordenamiento entre los genotipos, y el grado o magnitud de tal interacción, respectivamente.

Existió una alta concordancia en cuanto a la indicación de genotipos estables a través de las metodologías BLUP, IC, Annicchiarico (1992) y Lin y Binns (1988). En general, la metodología de modelos lineares mixtos permitiría aportar significativos antecedentes en la búsqueda de alternativas productivas de algodón con alta estabilidad en productividad de fibra, en los ambientes donde se pretende desarrollar su cultivo.

\section{Resumen}

Se analizó la predicción del efecto de cultivar vía mejor predicción lineal no sesgada (BLUP), de 14 cultivares de algodón (Gossypium hirsutum) establecidos en diferentes localidades de Brasil y Paraguay, en la temporada 20032004. BLUP, del rendimiento de cultivares, se comparó con el análisis clásico de estabilidad de los modelos desarrollados por Plaisted y Peterson, Wricke, Annicchiarico y Lin y Binns. Un abordaje Bayesiano a través del algoritmo de cadena independiente (IC), se utilizó como otra medida de comparación. La razón de verosimilitud evidenció diferencias significativas para los efectos de cultivar e interacción genotipo-ambiente. Los criterios de información de Akaike y Bayesiano confirmaron estos resultados. La mayoría de las correlaciones de Spearman y Pearson no fueron significativas sitio a sitio ( $\mathrm{p}>0,05)$, cuyos valores ( \pm error estándar) variaron de $-0,037 \pm 0,307$ a $0,565 \pm 0,259$ y de $-0,228 \pm 0,336$ a $0,604 \pm 0,257$, respectivamente. Los métodos de Plaisted y Peterson, y Wricke mostraron correlaciones no significativas con BLUP, IC, Annicchiarico, y Lin y Binns. Por otro lado, los coeficientes de Spearman fueron altos y significativos $(\mathrm{p}<0,01)$ entre BLUP y Annicchiarico: 0,859 $\pm 0,151$, y BLUP y Lin y Binns: $-0,899 \pm 0,099$. BLUP e IC, basado en los valores promedio y mediana a posteriori, evidenciaron idénticos ordenamientos de cultivares. CD406 y CD992221 fueron los cultivares que evidenciaron una estabilidad superior, confirmada por BLUP, IC, Annicchiarico y Lin y Binns, indicando una significativa concordancia entre los procedimientos. Metodología de modelos lineales mixtos permitiría la obtención de importante información genética de los cultivares de algodón con elevada productividad y estabilidad, en los ambientes considerados para su cultivo.

Palabras clave: BLUP, estabilidad de cultivares, Gossypium hirsutum, interacción genotipo-ambiente, mejoramiento, modelos mixtos, verosimilitud.

\section{Agradecimientos}

Los autores agradecen a la Cooperativa Central Agropecuaria de Desarrollo Tecnológico y Económico,COODETEC, delEstadode Paraná, Brasil, por el financiamiento de la presente investigación. El primer autor también agradece a la Coordinación de Perfeccionamiento del Personal de Nivel Superior (CAPES), del Ministerio de Educación de Brasil, por el financiamiento de beca doctoral.

\section{Literatura citada}

Annicchiarico, P. 1992. Cultivar adaptation and recommendation from alfafa trials in Northern Italy. Journal of Genetic and Breeding 46:269278.

Astorga, M. y F. Mora. 2005. Componentes de varianza e interacción variedad-sitio del vigor, producción y productividad de Olea europaea, en Chile. Cerne 11:25-33. 
Atroch, A.L., A.A. Soares, and M.A.P. Ramalho. 2000. Adaptabilidadee estabilidade delinhagens de arroz de sequeiro testadas no Estado de Minas Gerais. Ciência e Agrotecnologia 24:541-548.

Canechio-Filho, V., S.M.G. Passos and A. Jose. 1972. Principais culturas. Campinas: Instituto Campineiro de Ensino Agrícola, p.1-97.

Contreras, S. y C. Krarup. 2000. Interacción genotipo por ambiente en cinco cultivares de espárrago (Asparragus officinalis L.). Ciencia e Investigación Agraria 27:133-139.

Cruz, C.D. 2001. Programa Genes: Aplicativo Computacional em Genética e Estatística. Ediciones Universidade Federal de Viçosa. Viçosa, MG, Brasil. 648 pp.

Cruz, C.D. and A.J. Regazzi. 2001. Modelos biométricos aplicados ao melhoramento genético. Segunda edición. Ediciones Universidade Federal de Viçosa. Viçosa, MG, Brasil. 390 pp.

Elias, H.T., S. Hemp, C.A. Scapim, M.A. Rodovalho, M.R. Royer, F. Mora, and R.R. Barreto. 2005. Stability analysis of common bean genotypes in Santa Catarina State. Acta Scientiarum Agronomy 27:623-628.

Farias, F.J.C., M.A.P.Ramalho, L.P. Carvalho, J.A.N. Moreira, and J.N. Costa. 1997. Parâmetros de estabilidade propostos por Lin e Binns (1988) comparados com o método da regressão. Pesqui. Agropecu. Bras. 32:407-414.

Henderson, C.R. 1973. Sire evaluation and genetic trends, p.10-41. In: Proc. Animal Breeding and Genetic Symposium in Honor of Dr. J.L. Lush. Champaign, IL, USA.

Henderson, C.R. 1984. Applications of Linear Models in Animal Breeding. University of Guelph. Ontario, USA. 462 pp.

Lin, C.S. and M.R. Binns. 1988. A superiority measure of cultivar performance for cultivar $\mathrm{x}$ location data. Can. J. Plant Sci. 68:193-198.

Littell, R.C., G.A. Milliken, W.W. Stroup, and R.D. Wolfinger. 1996. SAS System for Mixed Models. SAS Institute Inc., Cary, North Caroline, USA. $633 \mathrm{pp}$.
Martins, E.N., P.S. Lopes, M.A. Silva, and R.A.A.T Junior. 1997. Uso de Modelos Mistos na Avaliação Genética Animal. Ediciones Universidade Federal de Viçosa. Viçosa, MG, Brasil. 121 pp.

Mora, F. y A. Arnhold. 2006. Inferencia Bayesiana y metodología de modelos lineales mixtos aplicados al mejoramiento del maíz. Ciencia e Investigación Agraria 33:217-223.

Moresco E.R. 2003. Progresso genético no melhoramento de algodoeiro no Estado de Mato Grosso. Tesis de Doctorado, Universidad de Sao Paulo, Piracicaba, Brasil. 79 pp.

Plaisted, R.L., and L.C. Peterson. 1959. A technique for evaluating the ability of selections to yield consistently over locations or seasons. American Potato Journal 36:381-385.

Perri, S.H.V., and A.F. Iemma. 1999. Procedimento MIXED do SAS® para análise de modelos mistos. Scientia Agricola 56:959-967.

Piepho, H.P. and J. Möhring. 2006. Selection in Cultivar Trials - Is It Ignorable?. Crop Science 46:192-201.

Resende, M.D.V., E. Furlani-Junior, M.L.T Moraes, and L.C. Fazuoli. 2001. Estimation of genetic parameters and prediction of genotypic values in coffee breeding by the REML/BLUP method. Bragantia 60:185-193.

Scapim, C.A., V.R. Oliveira, A.L. Braccini, C.D. Cruz, C.A.B. Andrade, and M.C.G. Vidigal. 2000. Yield stability in maize (Zea mays L.) and correlations among the parameters of the Eberhart and Russell, Lin and Binns and Huehn models. Genet. Mol. Biol. 23:387-393.

Wricke, G. 1965. Zur Berechnung der Ökovalenz bei Sommerweizen und Hafer. Pflanzenzuchtung 52:127-138.

Yang, R.C. 2002. Likelihood-Based Analysis of Genotype-Environment Interactions. Crop Science 42:1434-1440.

Yue, G.L., K.L. Roozeboom, W.T. Schapaugh, and G.H. Liang. 1997. Evaluation of soybean cultivars using parametric and nonparametric stability estimates. Plant Breed. 116: 271-275. 